\title{
The use of geomatic techniques to improve the management of metro infrastructure
}

Ma Amparo Núñez-Andrés ${ }^{1)}$ Felipe Buill ${ }^{1)}$ Saturio Delgado-Medina ${ }^{2)}$ Carlos Plancho-Milian ${ }^{3)}$

1) Department of Civil and Environmental Engineering. Division of Geotechnical Engineering and Geoscience, Universitat Politècnica de Catalunya

2) Unitat de projectes d'infraestructura i via, Ferrocarril Metropolita de Barcelona

3) Infraestructuras, COMSA SAU

The management of large infrastructures requires comprehensive knowledge of their general characteristics and the elements that constitute them. It is essential to know which elements are involved and where they are located. At the same time, it is vital to understand the overall geometry. The accuracy and precision required for both types of information is different: much greater accuracy is required in the understanding of the geometry. However, it is difficult to obtain this information about underground infrastructures due to their lighting characteristics, hazards, lack of GNSS (Global Navigation Satellite System) signals, and time available. In this case, to achieve the required quality in both tasks, the mobile mapping systems that are widely used in large surveys and urban inventories have to be adapted to obtain data in underground conditions. To achieve this, considerable geodesic support is necessary. The GNSS positioning system should be replaced by IMU sensors, and infrared sensor cameras are used, among other pieces of equipment. To establish the trajectory, additional information extracted from the targets detected in the laser scanning point clouds must be used. Therefore, a laser scanning and kinematic photogrammetry survey was carried out to obtain the centimetric precision and complete information that is still used today. This paper presents an adaptation of these systems for use in the Ferrocarril Metropolità de Barcelona.

Keywords: mobile mapping, photogrammetry, underground surveying, infrastructure, management transport infrastructure, laser scanning.

\section{Introduction}

Large infrastructures are present throughout Spain. When the need arises, construction projects are undertaken to build large infrastructure, but they do not always take into consideration the planning and subsequent management of maintenance. 
In some cases, it is easy to carry out this management. However, in other cases, such as the underground network, it may be much more difficult, since this is a complex system in which everything must function so that passengers, and consequently city life, are not affected. For effective management, it is vital to know all the elements that are part of the Metropolitan Railway's different systems. These systems are increasingly complex, with elements such as the grid, roads and signage, among others. In other words, we need an inventory of these elements, which must also be georeferenced.

The location and characteristics of these elements have traditionally been obtained independently and discreetly. For each type of system, technical experts have to move to the area and record the features, while its location is given in a survey. Consequently, a great number of people have to go into the tunnels, and there are many disadvantages in terms of time and safety. Moreover, the fact that this is not a massive data capture means that the experts must return to the tunnel to measure new points.

Existing software can be used to manage road infrastructure inventories (McCarthy et al., 2007), but not railway or underground infrastructure inventories. With the development of smart cities, examples such as Singapore have emerged, where transport network management is contained in an ISI (Infrastructure for Spatial Information), according to OGC (Open Geospatial Consortium) standards.

We face two difficulties: capturing all the spatial information that is required to carry out the inventory; and managing this information. The working conditions for the capture of spatial information should be considered. It is very difficult to obtain data using traditional geomatics techniques in underground tunnels, not only because of the nature of the network and its confined and limited spaces, but also because of the continuous activity that takes place in the tunnels. 
Traditional topographic methods used to detect geometric changes in the design of the railway are very common, but they only allow us to obtain data on a limited number of elements. Consequently, it is vital to find geomatics solutions to obtain data on a large number of objects, with enough quality to be georeferenced in a short time. Currently, the techniques employed in the mass capture of spatial information are kinematic and static photogrammetry and laser scanning, using GNSS (Global Navigation Satellite System) positioning systems. Examples are found in Kingston et al. (2007) and Talaya et al. (2007). Working inside galleries makes positioning difficult, so the support of other sensors and indirect georeferencing is essential.

This is another of the challenges to be solved: while georeferencing of continuous capture is precisely resolved in surface work through differential GNSS systems, in underground work or in closed environments methods must be developed that allow georeferencing of the trajectory with sufficient precision, since the drift that inertial measurement unit (IMU) suffer makes it impossible to use the individual form for this task.

In the following sections, we analyse how this task was carried out to obtain a network inventory of the Ferrocarril Metropolità de Barcelona (FMB), managed by Transports Metropolitans de Barcelona (TMB) using a Mobile Mapping System (MMS) adapted to the underground conditions.

This solution has three main advantages:

- $\quad$ Field work can be performed quickly and elements can subsequently be identified in the office by specialists in each field.

- Due to the availability of tools, data can be continuously extracted and the results validated. 
- $\quad$ Safety is increased, since the flow of people circulating through the tunnels is reduced.

\section{D acquisition techniques}

Usually, geospatial information for civil works is obtained by traditional topographic methods, from bases that belong to a geodetic network. Detailed information is captured by radiation, with laser scanning, aerial or terrestrial methods, and GNSS receivers for satellite data (Velasco et al., 2014) or air and terrestrial photogrammetric methods.

When the work is carried out in tunnels, fewer suitable methods are available, and it is difficult to obtain redundant information. In topography and photogrammetry, the problem is solved using specific instruments and methods (Wang et al., 2009). In the case of kinematic, photogrammetric or laser scanners, systems must be used to check the distances and turns of the sensors used, for example odometers and inertial systems (INSs) (Gräfe, 2007). New sensors have now been incorporated to complement these traditional methods and ensure faster, complete, dynamic geospatial data capture. These are known as mobile mapping systems (MMSs).

The traditional system is not valid in an underground environment, since positioning and subsequent georeferencing of the trajectory through differential GNSS systems cannot be used, as no signal is received inside the tunnel. In these cases, the IMU must be replaced by a more accurate device with minimum drift, which makes the system more expensive. In addition, external signals are required that allow us to recalibrate the system once the deviation of the path has reached the allowed tolerance.

\section{Photogrammetry}

Photogrammetry has been widely used to obtain mapping information for nearly 100 years. The most common method is the use of photographs taken from aerial platforms with 
appropriate geometry for the subsequent work of staff and adequate lighting conditions (Slama et al., 1980). The terrestrial method is used to obtain the geometry of unmapped surfaces. In this case, cameras are placed in fixed positions over the ground. A special method involves the use of a mobile platform that moves along the Earth's surface obtaining photographs with an angle of inclination relative to the vertical that may be considerable. This is known as the kinematic method (Scaioni et al., 2014) (Dai and Peng, 2013).

Photogrammetry or videogrammetry has been successfully used for over 20 years to capture or update information on highways or railways and for infrastructure inventories. One example is the Geovista application, which uses multiple cameras from platforms such as GeoVan (Talaya et al., 2007).

A study of different techniques to capture spatial data from cameras can be found at Zhu and Brilakis (2009), where the pros and cons of each technique are discussed. The main purpose of this study is to select the proper technique based on the application requirements of the data infrastructure. Cameras on mobile platforms are used not only to capture $3 D$ information, but also to detect problems in the pavement (cracks and potholes), thus reducing the consumption of time and resources (Koch and Brilakis, 2011).

The type of spectral sensitivity used by the sensors in these cameras changes depending on the objective of the work. The panchromatic sensor or separate $R G B$ is the most commonly used system, and in some cameras the near infrared channel (NIR) is also used. In interior work, short-range lighting systems that allow good light for capturing images can be used. In this case, sensitive cameras in the spectrum of infrared can be employed with illumination of the same type. However, the use of long wavelength, NIR, is inversely related to the resolution and the separator of the objectives: at longer wavelengths, the spatial resolution power is lower (Slama et al., 1980). 
One of the challenges that we face in taking photographs in tunnels is a lack of light. Therefore, in these cases, artificial lighting (preferably LEDs) was required in addition to infrared sensors. The disadvantage is that these cameras have a much lower resolution of below 1.5 Mpx than conventional sensors.

\section{Laser scanning}

Terrestrial laser scanning (TLS) is widely used for numerous applications, among them infrastructure management and monitoring. In tunnels, it can be very useful to obtain elements to make management easier (Yoon et al., 2009), in geometry extraction (Gikas and Stratakos, 2012; Seo et al., 2008; Han et al., 2013), and in deformation analysis of vaults and walls (Wang et al., 2009), among other tasks.

This technique is suitable for many applications, since it allows us to capture 3D information without direct contact with the object. Centimetre accuracy is obtained in a very short time, and in unfavourable conditions.

In most applications of subterranean surveys, for example in caves, mines or tunnels, the laser head is placed on a tripod. Several studies examine factors that influence accuracy and precision, such as the distance between the sensor and the object, the type of material and, in the case of a tunnel, the angle of incidence (Pejic et al., 2013; Roca-Pardiñas et al., 2014).

Specific conditions affect work in a tunnel: the working distance is more limited, the atmospheric conditions influence the reflectivity of the material and are not controlled (Pejic et al., 2013), the work is basically in a line, and the localisation of the TLS stations is critical to the analysis, in order to avoid incidence angles that lead to intolerable errors. Pejic et al. (2013) determined that the influence of the angle of incidence on measurement noise is 30\%, so in the normal conditions of a railway tunnel, the maximum distance scan should be $13 \mathrm{~mm}$, 
which significantly increases the number of stations required. The ideal localisation of the TLS is the centre of the tunnel, in order to distribute the noise evenly. If the sensor is near the gable, the errors are not uniform in cross-section, and are higher in the nearest wall due to the angle of incidence (Roca-Pardiñas et al., 2014).

To improve the capture/time ratio, the system is used in kinematic mode and must be integrated with other systems. This process is explained in more detail in the following section.

In static or kinematic modes, control points, which are usually targets, are required to georeference the point cloud. The coordinates are obtained by topographic methods and their accuracy contributes directly to the accuracy of the final model (Pejic et al., 2013; Puente et al., 2013).

\section{Mobile mapping systems}

The need to increase the productivity of laser scanner sensors and their application to urban inventories and to control pavements, among other factors (Jacobs, 2005; Kingston et al., 2006), has led to their use in kinematic mode. While some of the initial applications were a 'stop-and-go' system, in which the sensor was on a moving platform but stopped at the time of capture, due to integration with other systems, data collection while fully in motion has gradually become possible.

To achieve this, most systems are fixed to a stable platform. This is then attached to the vehicle's roof where the cameras are integrated with the TLS systems and GNSS antennas. The car is also equipped with odometers and INSs. The distribution of the components varies depending on the application, but in any case the set has to be calibrated previously to the data capture (Gräfe, 2007). 
The differential GNSS allows positioning with absolute centimetre quality. The positions are determined for two main reasons: to georeference the point cloud, and initialise the $I M U$ when its bias exceeds the stated tolerance. Likewise, the position from the inertial sensors is used when the GNSS signal is lost by occlusion, as long as it is for a short period of time only.

As mentioned above, the use of this kind of positioning systems in mines, caves and tunnels is not practical. It is essential to set control points, patterns, or mixed systems that allow underground positioning. One of the latter systems consists of replacing TLS sensors with 3D ToF (Time of Flight) cameras (Ratshidaho et al., 2014).

\section{Barcelona underground management}

At the time of this study, the metro network in Barcelona comprised $173 \mathrm{~km}$ of commercial railways divided into 7 lines. The network has now been extended to $208 \mathrm{~km}$ and 9 lines. The proper management of all elements in this underground network is based on the knowledge of their location and characteristics. Therefore, with the aim of improving management, TMB worked on a pioneering project in Spain, which had two main objectives:

- Creation of a cartographic base. Establishment of a geodetic network that allows georeferencing, topographic surveying of the geometry and geometric design calculation. Subcentimetric accuracy was required in the geometric determination of the railway and the geodetic network.

- Inventory of the different elements (signalling, communication and sidewalks), to record both their location in absolute coordinates and their attributes. In this case, the accuracy required in the coordinates of the different elements was in a submetric order, and therefore much lower than in the previous case. 
It was impossible to obtain this information in the traditional way for the entire network, so it was essential to find technology that could allow us to gather the data in a relative short time, taking into account that this work could not interrupt the passenger service or maintenance tasks. Therefore, we sought a system that could capture the information in the shortest possible time, with guaranteed quality (Fig. 1, bottom).

Under these conditions, the solution used to extract information in the tunnels was to carry out a kinematic survey, combining laser scanning and digital photogrammetry. This allowed us to inventory the elements in the office, and to obtain a $3 D$ laser scanner model and a photogrammetric model of the tunnels.

The elements to be inventoried could be classified into five groups: railroads, works, catenary, energy and signalling.

- Railroad: includes all the characteristic elements of the railroad, types of fixation, sleepers, seat type, oilers, detours, expansion devices and molehills.

- Works: includes all elements relating to infrastructure, types of vault, ventilation shafts and platforms, changes in section, bridges and walkways.

- Signalling: includes signals, track circuits, signalling cabinets and elements of communication, changes and drives, ATP (Automatic Train Protection) and ATO (Automatic Train Operation) elements.

- Catenary: comprises supporters, types of catenary insulators and switches.

- Energy: substations, switching cameras, transformer centres, and points of light, feeders and power lines are identified in this category. 

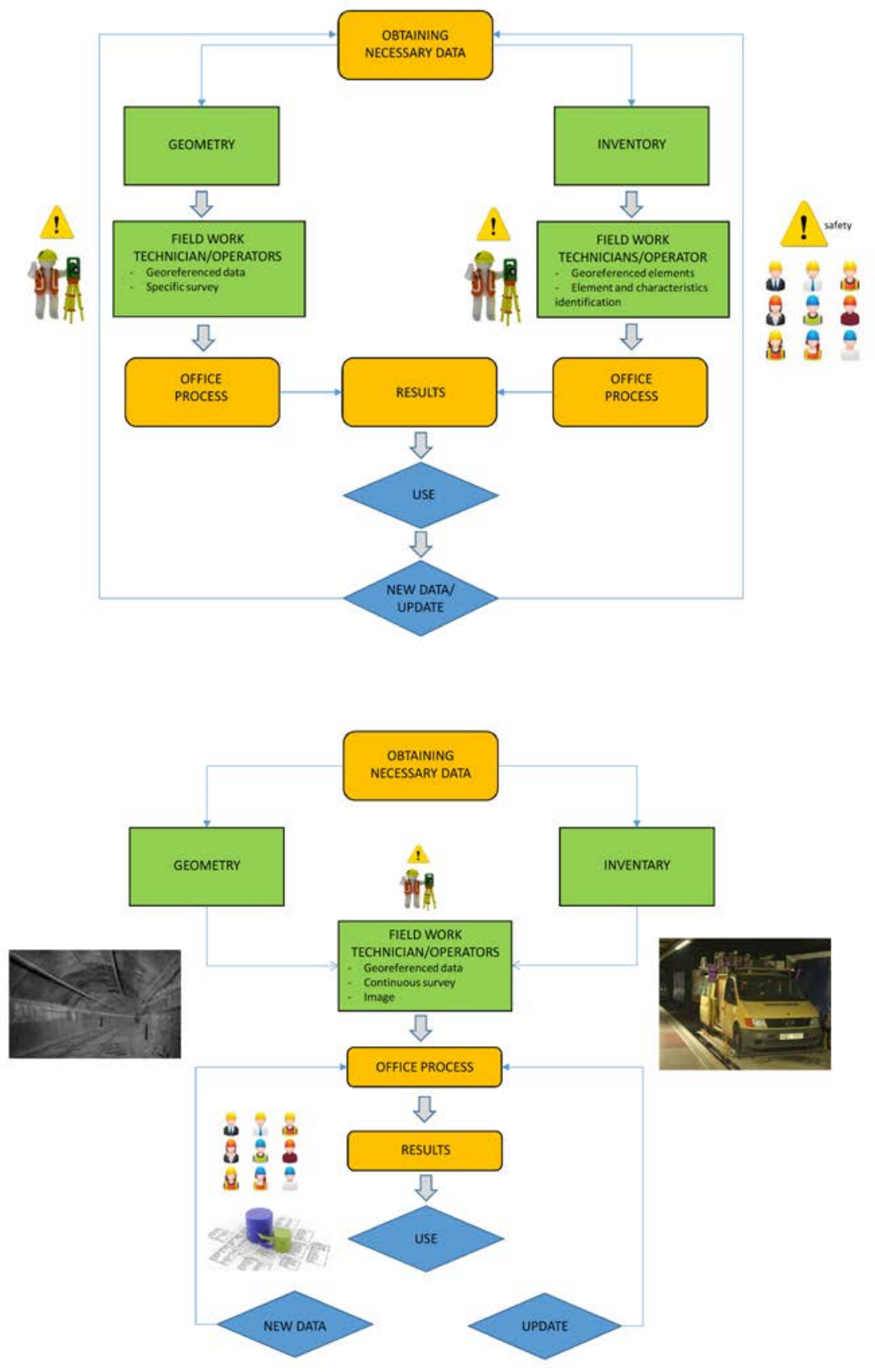

Fig 1. Flow chart of the work and people involved before and after of the methodology change

Within each group subcategories were defined, and specialised technical assistance was required to identify and classify the elements.

Before this project (Fig. 1, top), georeferenced data on any elements of tunnels and stations could be obtained only with a topographic survey, which involves time-consuming tasks to connect with coordinate frame systems. This is a disadvantage when little time is 
available. Moreover, the equipment that is used is extremely specialised, which means that the work must be done by the specific technicians who can operate each piece of equipment, as well as by the geomatic team who georeference the data. In other words, the work is slow and requires a lot of human resources.

As a result of the project presented here, a continuous record of the continent and content of the tunnels and part of the TMB stations was obtained. These data were gathered quickly and enabled us to extract information a posteriori, even years after the data acquisition. Therefore, each technical specialist could extract the required information in the office with no time pressures, and could compare data and consult the model and photographs when new information was required.

\section{Methodology}

The MoSES mobile sensing system (Gräfe, 2007) was chosen for the data capture. This system had an original configuration to work in open air zones, based on four modules:

- Trajectory module: an IMU Applanix POS / LV with a bias from 1 to $0.01^{\circ}$ per hour, recalibrated with the integrated GPS system and an odometer.

- Photogrammetric module: composed of six colour cameras with the sensitivity required to work in daylight.

- Laser-scanner module: two sensors Sick LMS200 ToF, with $180^{\circ}$ coverage and nadir orientation to obtain pavement data.

- Video module: to film in the forward direction. 

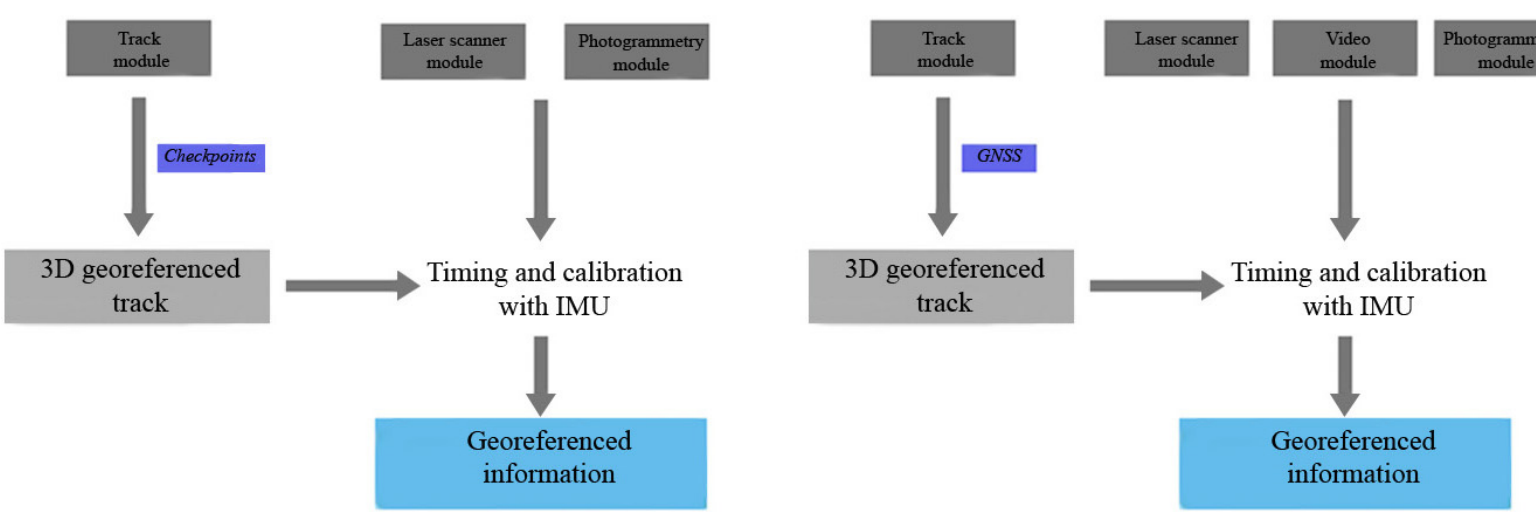

Fig 2. Schedule of the modules modification

To work in the tunnel conditions, i.e. poor lighting and no GPS coverage to recalibrate the IMU, the system had to be modified in the modules of trajectory, photogrammetry and laser scanning (see Fig. 2).

The set of cameras, laser scanners, odometers and kinematic systems were calibrated in a controlled environment (calibration field), consisting of a set of control targets located on the floor and on the facades of the inspection area. From an initial approximate calibration, the data of the sensors (cloud of points and images) and the control points were measured.

Successively the corrections were applied in a process of adjustment by least squares, until a solution with the required precision was reached. The final result is a set of data including the internal data of the cameras and the orientations of the sensors with respect to the INS.

Various tests were carried out to calibrate the measurement systems. For this, three types of reference distances with different orientations were used, captured simultaneously with MoSES. All these were carried out in high accuracy test fields for the complete equipment (cameras and video, laser scanner, odometer and $I M U$ ).

Working without GPS coverage affects the georeferencing trajectory. In this case, as 
mentioned in Section 2.3, control points are required to correct the bias of INSs. These targets are reference plates, $P K$ (Point-Kilometric), located on gables, whose absolute coordinates are obtained from the geodetic control network. These points were separated by $20 \mathrm{~m}$, which is a sufficient gap, according to the results of previous studies (Puentes et al., 2014).

Therefore, we have two work methods, depending on the final result to be obtained. However, both methods share common processes, such as the trajectory definition. Figure 3 reflects the workflow, in which the sensors used to capture the $3 D$ information and to define the trajectory are differentiated.

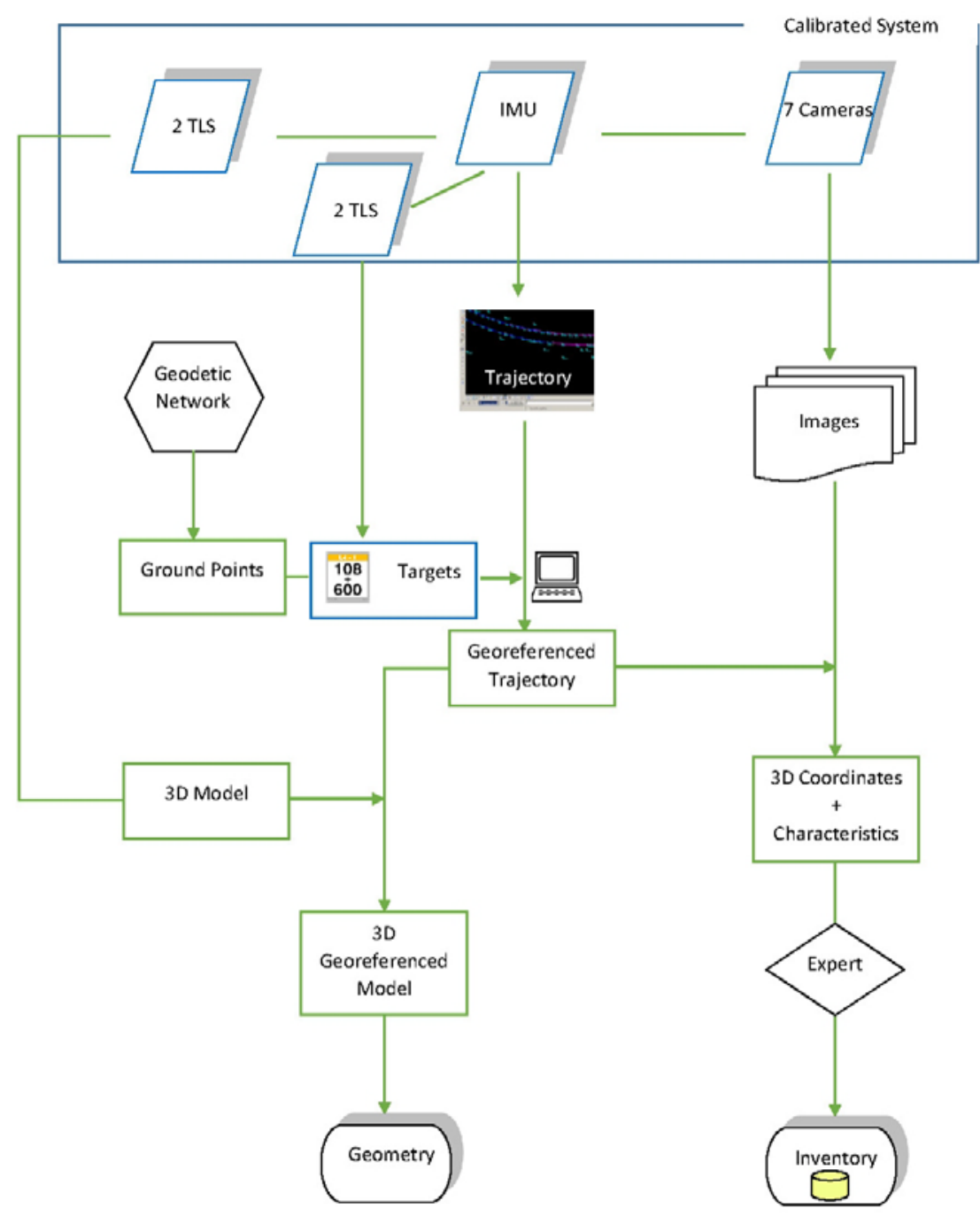

Fig 3. Flowchart of the trajectory, photogrammetry and laser scanning modules and their integration. In red the sensors used in the trajectory definition and in green the sensor whose aim is the data capture 
To achieve high accuracy and precision geometry and scanning in a tunnel requires an adequate geodetic network, in order to register and georeference the point clouds and check the scan data quality. Therefore, one of the main, first tasks is to establish a reference frame.

\section{Reference system}

Owing to the kind and conditions of the work to provide coordinates for targets used in georeferencing, three networks were required, each supported in the higher order, as shown in Fig. 4.

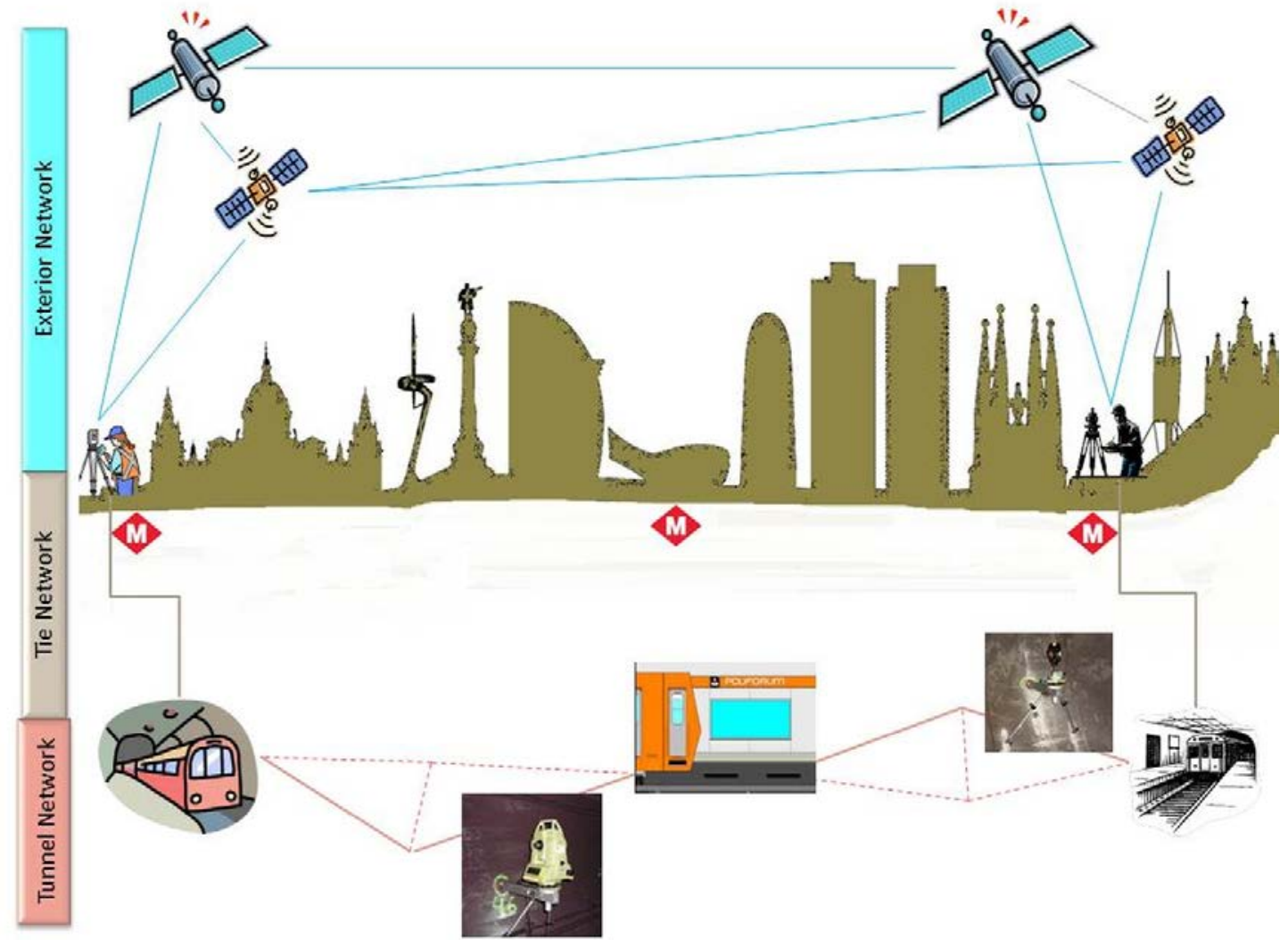

Fig 4. Control geodetic network (exterior, tie, and interior) in vertical section

The Exterior Network is the connection to the geodetic reference frame of Catalonia. It consists of 136 vertices, 124 of them at the entrances of the stations and garages chosen as the start and end of the Tie Network, and 12 belonging to the $X U$ (Xarxa Utilitaria, 4th order network) of the ICGC (Institut Cartogràfic i Geològic de Catalunya), distributed 
homogeneously over the study area, Fig. 5. This network was observed by static GPS methods. Each base was observed 4-7 times; hence 982 baselines were available with enough redundancies to achieve the required accuracy. After the network adjustment, the coordinates were obtained with an average standard deviation of $2 \mathrm{~cm}$ in planimetric and altimetric components.
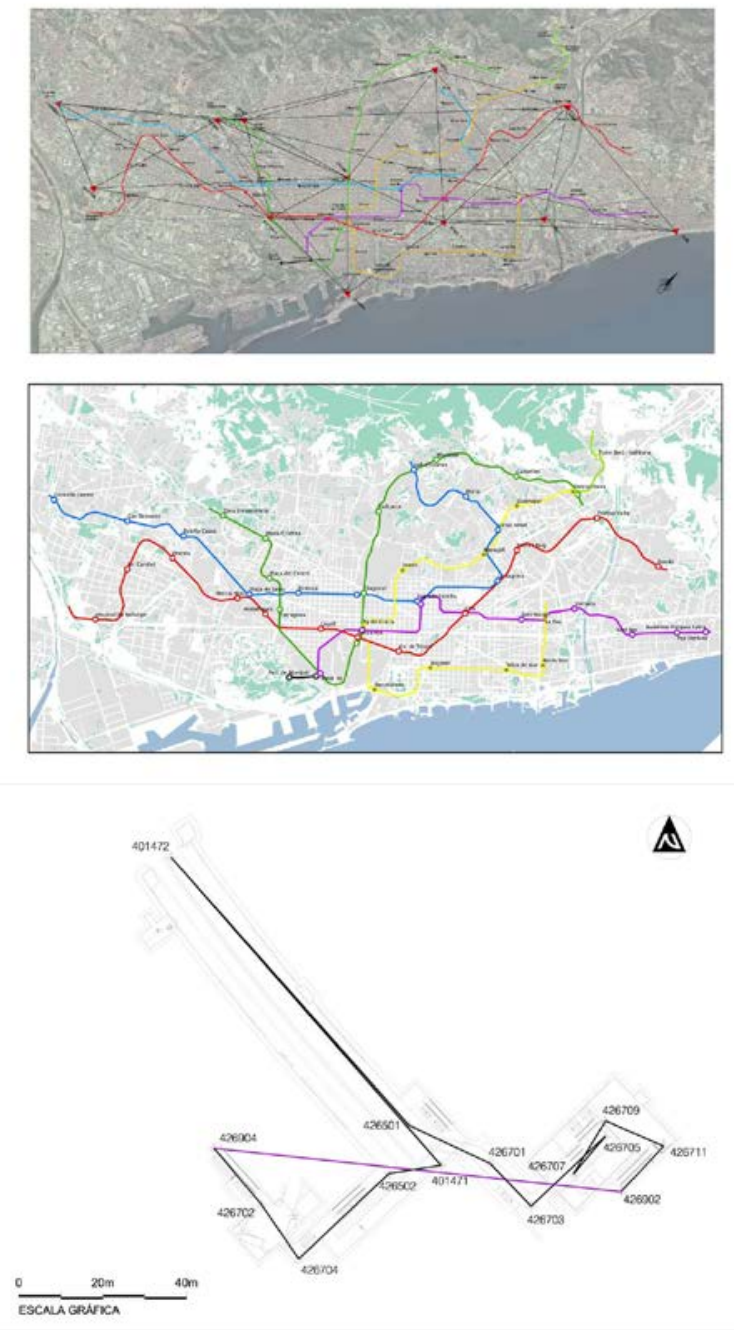

Fig 5. Exterior geodetic network, interior network and an example of a tie network in one of the stations

The Tie Network is comprises of the traverses that connect the Exterior and Interior Networks. In total, 53 traverses have been observed, consisting of 894 vertices. After adjustment by least squares, coordinates were obtained with an average RMS of $1 \mathrm{~cm}$. 
The Interior Network comprises a main traverse along the tunnel connecting two consecutive Tie Networks, and the radiated bolts from the bases. There are a total of 73 traverses formed by 883 vertices and 3522 bolts. In this case, the average RMS obtained or the coordinates after adjustment is $1 \mathrm{~cm}$.

Interior and Tie Networks were observed under unfavourable conditions. Observations of the Interior Network must be made inside the tunnels and are affected by factors such as lateral refraction (Velasco-Gómez et al., 2016). Observations of the Tie Networks are performed within the stations, so the geometry is very unfavourable. To try to minimise the errors in these conditions, high-precision surveying equipment and forced centring systems were used. The method used for observations and calculations included three series on I and II reciprocal observations angle and distance, and collimations using ATR (Automatic Target Recognition). Thus, erroneous observations could be filtered before proceeding to the calculation. Both networks were adjusted by the least squares indirect observations method, applying the $W$-Baarda test (Baarda, 1967) for detecting gross errors, and setting $\alpha$ and $\beta$ at $0.1 \%$ in $80 \%$.

\section{Laser scanning}

The initial configuration of the laser scanning module was replaced by two phase difference sensors, $Z+F$ Imager 5006 and FARO LS880, since the optimal maximum range is different for each one, to obtain the $3 D$ model. The original sensors, Sick LMS200, were used to reference the trajectory, because they are positioned to capture $P K$ targets.

In order to capture the data, the truck was assembled on a platform driven by a draisine used in the maintenance tasks of TMB.

The survey took place during the time slot reserved for maintenance tasks, between 
1:00am and 4:15am, when the catenary has no voltage. Considering the time required to start up the system and transfer the draisine from the garage to the starting point and vice versa on completing the work, only 2 hours were available for the data capture. The system allowed us to obtain data at $10 \mathrm{~km} / \mathrm{h}$, which is a profile every $5 \mathrm{~cm}$ (Fig. 6), so that each day between 15 and $20 \mathrm{~km}$ of infrastructure were measured. The complete survey was finished in 10 working days.

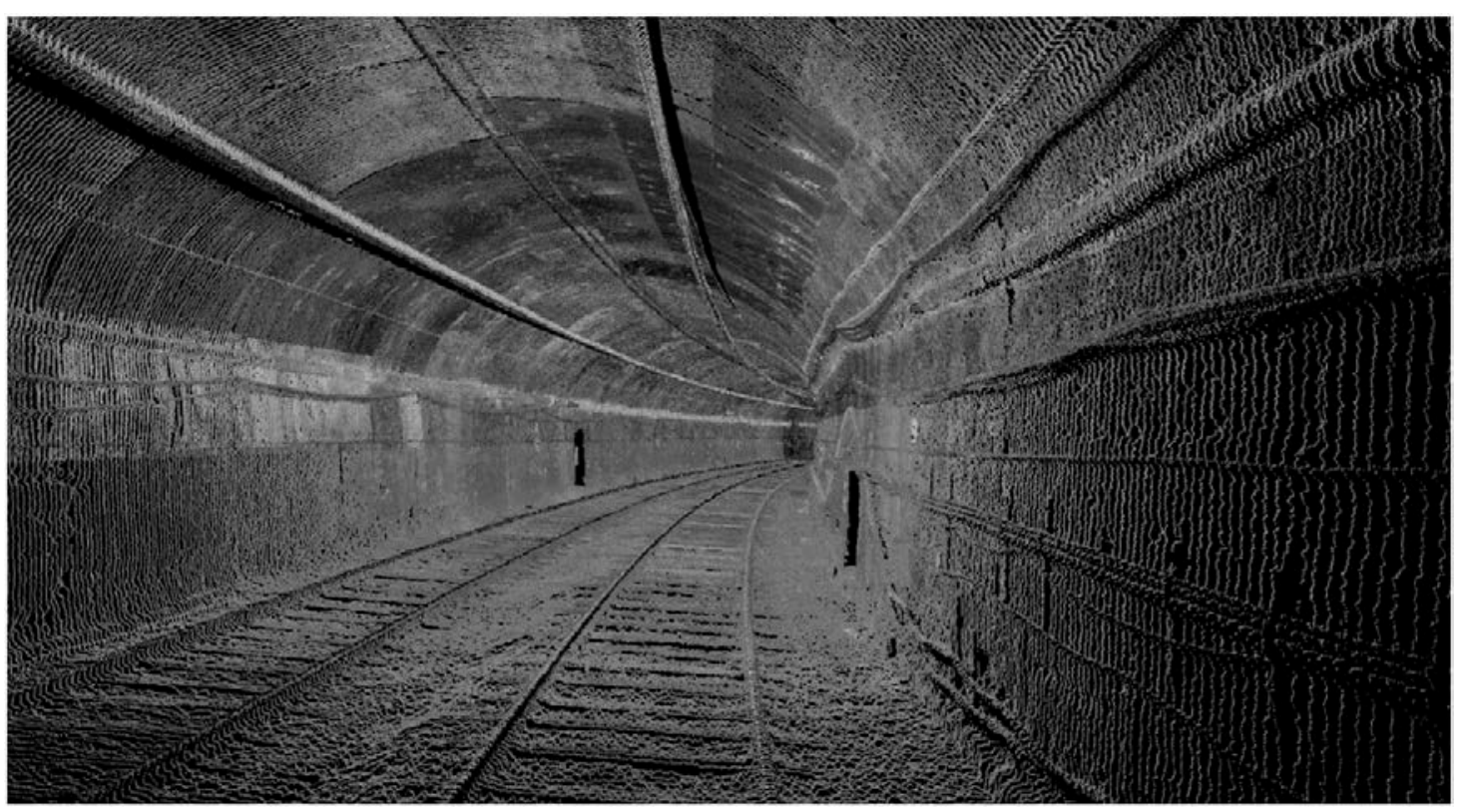

Fig 6. Tunnel georeferenced section

After these tasks, partial cloud points were filtered and registered to obtain a complete cloud of the tunnel, formed by 13809 million points.

\section{Georeferencing}

Since it was impossible to obtain GNSS positioning to restart the IMU, the original position had to be replaced by another with greater trajectory accuracy. In this case, the IMAR iNAV$R Q H$ was used based on a laser gyroscope, with a bias from $0.01^{\circ}$ to $0.0015^{\circ}$.

The trajectory during the data capture was calculated by integrating data from the $I M U$, 
the odometer and the Kalman filter. This computed trajectory is continuous, in this case with a bias of $0.01^{\circ}$, which represents $17.5 \mathrm{~cm} / \mathrm{km}$. This should be corrected and georeferenced with the previously measured targets, which are located every $20 \mathrm{~m}$. The targets were identified in the data from oblique scanners, since they intersected all the edges of the plate. In this way, according to the bias estimated by the technical characteristics of the $I M U$, the method used to correct it and the number of control points used for this transformation, the final precision in the calculated trajectory was estimated at $\pm 2 \mathrm{~cm}$ and $\pm 5 \mathrm{~mm}$ in $X Y$ and $Z$, respectively. This can be checked empirically by comparison with the measures of the railway geometry obtained by topographic methods.

Once the trajectory had been determined, and since the whole system was calibrated, the sensor positions were known at all times, and therefore the georeferenced coordinates of all the data from scanning and cameras were also known, which facilitated the photogrammetric processes.

\section{Photogrammetric survey}

In the photogrammetric module, the six $R G B$ cameras needed to be replaced by seven high sensitivity infrared spectrum $J A I$ cameras with auxiliary LED lighting, which was also infrared, Fig. 7. Thus, a more uniform illumination was achieved and the contrasts caused by the spotlight used with $R G B$ cameras were avoided. In addition, the localisation and orientation of the cameras needed to be changed, so that they were focused on the gables, vault and floor of the tunnel. 


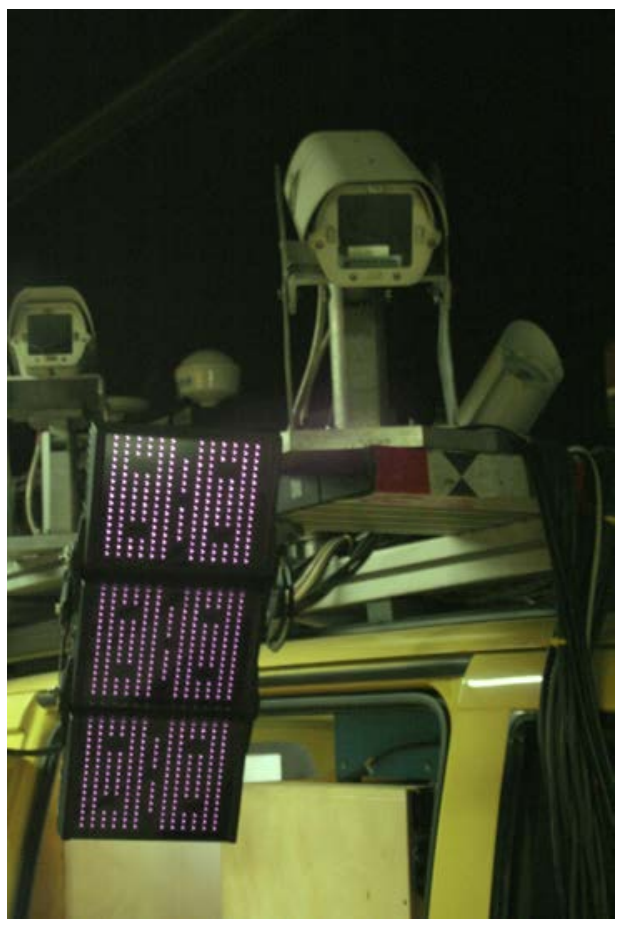

Fig 7. Infrared $L E D$ auxiliary lighting

The system was composed of a camera in almost zenith orientation, to capture images of the catenary; two cameras in quasi nadir orientation (formed stereoscopic pair), to capture images of the left and right rail profile respectively; two cameras in oblique orientation, to capture images of the middle-top of the gables on both sides of the vehicle; and finally two cameras in oblique orientation to capture the middle-bottom of the gables. Two types of cameras with focal $8 \mathrm{~mm}$ and $12 \mathrm{~mm}$ were used since the range camera-object is closed due to the tunnel characteristics. The sensor was of type 2/3", with a resolution of 1040x1392, less than the $R G B$, and a pixel size of 6.45 microns.

One of the difficulties to manage is the fact that the distances between object and camera were different for each one. They depended on the position of the camera in the system and the kind of tunnel. Moreover, the images taken from the side cameras were parallel to the tunnel. Depending on which part of the image was used for the measurement, the elements could be found to be a few centimetres or several meters.

In the case of photographs taken at a distance of $2 \mathrm{~m}$, the value of the GSD (Ground 
Sample Distance) obtained is slightly more than $1 \mathrm{~mm}$, reaching the edges of the photographs at $3 \mathrm{~mm}$, considering for this calculation a distance of $5 \mathrm{~m}$ and a focal $12 \mathrm{~mm}$ (values between 1/167 and 1/417). For a focal length of $8 \mathrm{~mm}$ these values are increased by $50 \%$ (Eq. 1).

$$
\mathrm{E}=\frac{\mathrm{D}}{\mathrm{f}} \quad(\mathrm{Eq} .1)
$$

For a distance $(D)$ of $2 \mathrm{~m}$, the scale would reach a value of $1 / 250(1 / E)$, and if the distance is increased to $5 \mathrm{~m}$ the scale would be 1/625 (1/E), and the corresponding values of GSD (GSD $=\mathrm{E} \cdot$ pixel) are $2 \mathrm{~mm}$ and $5 \mathrm{~mm}$, respectively (1/E= photographic scale).

In any case, the cameras enabled millimetric precision to be obtained in the measurement of a point, and therefore the precision was higher than that in the topographic work. As a consequence, the final precision was given by the topographic vertex, in accordance with the established tolerances, i.e. it depends basically on the quality in the topographic vertex, which is in accordance with the established tolerances.

Since the survey is in a kinematic mode, the photogrammetric pair could be from images taken at the same time from different cameras if the images have an overlapping area, or from images taken from the same camera at different times (example: $t=1$ and $t=2$ ). However, in this last case, the quality is lower.

With these photographs and the photogrammetric data processing programme, the spatial coordinates of details can be established from the stereoscopic pair or from monoscopic photographs and the laser model (see Fig. 8). 

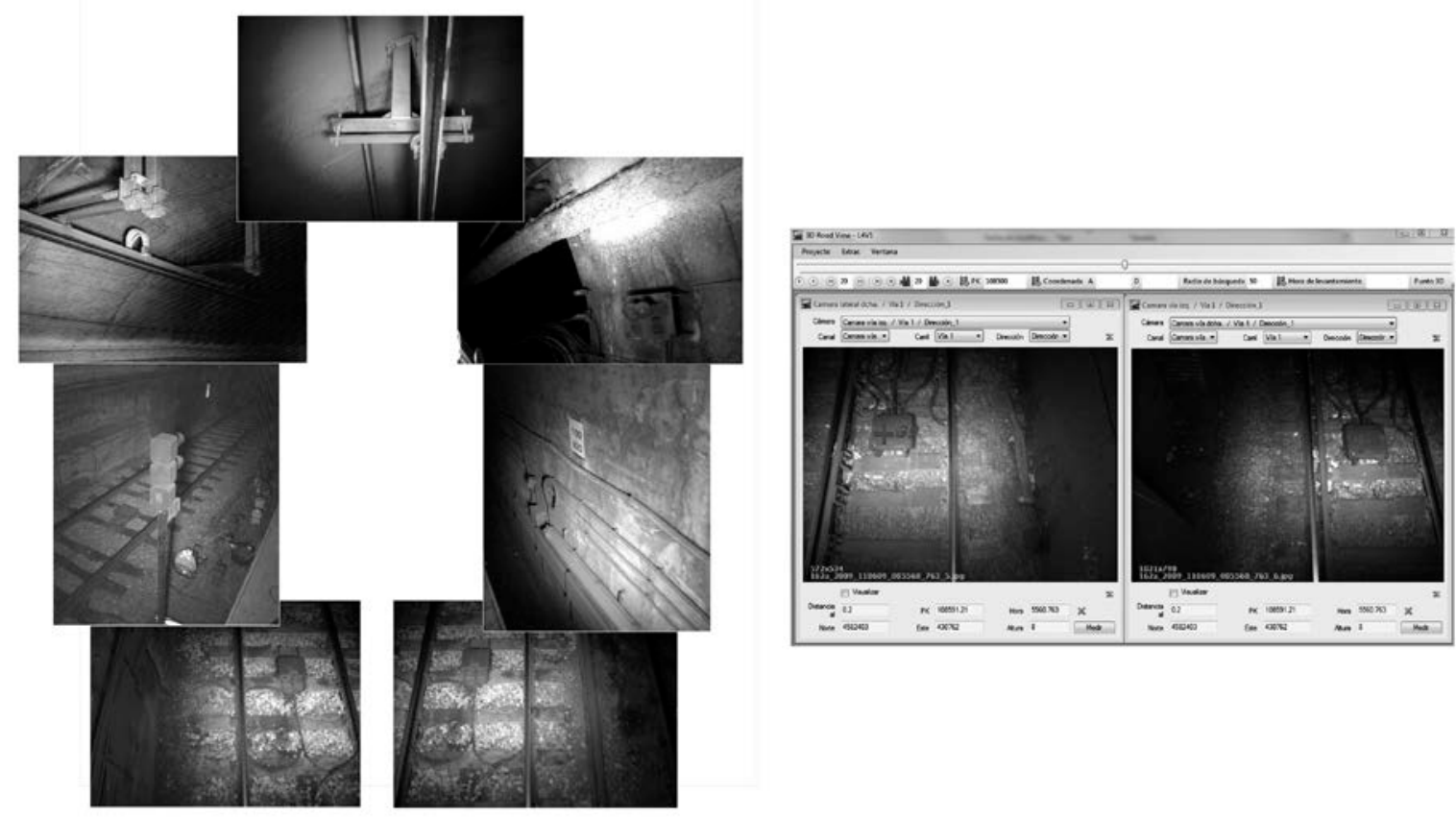

Fig 8. Photograms from the7 cameras used in the photogrammetric process and measured point in two photography's obtained in the same time by two cameras

The error in the determination of the coordinates of a point is lower than the estimated in the camera position, for example, the side of the tunnel.

Considering the parallax equations for obtaining the ground coordinates (Eq. 2)

$$
\mathrm{X}=\mathrm{E}_{\mathrm{f}} \cdot \mathrm{x} \quad \mathrm{Y}=\mathrm{E}_{\mathrm{f}} \cdot \mathrm{y} \quad \mathrm{Z}=\mathrm{E}_{\mathrm{f}} \cdot \mathrm{f}=\frac{\mathrm{B}}{\mathrm{p}} \cdot f
$$

So, for any orientation the planimetric error is expressed by Eqs. (2) and (3).

$B=$ Photographic base $p=$ parallax $f=$ focal length $\quad E_{f}=$ photographic scale

$$
d X=E_{f} \cdot d x \quad d Y=E_{f} \cdot d y \quad d x=d y \quad d X Y=\sqrt{2} \cdot E_{f} \cdot d x(E q .3)
$$

And in depth by Eq. 4, where the error in the parallax is shown in Eq. 5.

$$
\begin{aligned}
& d Z=\frac{Z \cdot Z}{B \cdot f} \cdot d p=\frac{Z}{B} \cdot E_{f} \cdot d p \\
& d p=\sqrt{2} \cdot d x
\end{aligned}
$$

In the case of study for the worst value of $E_{f}$ the planimetric error would be $6 \mathrm{~mm}$ and in 
depth $28 \mathrm{~mm}$. So, when we change from the photographic system to the cartographic one, we obtained the following: $\mathrm{dEN} \approx 28 \mathrm{~mm}, \mathrm{dH} \approx 5 \mathrm{~mm}$, perfectly in the range of the tolerance previously established.

As this is a kinematic survey, photogrammetric methods for calculations can generate pairs of images obtained at the same time from different cameras and different moments with only camera (Fig. 8).

As result, a $3 D$ model of all FMB tunnels was obtained. This has enough definition to identify and locate any registered item, so is the basis for the inventory.

\section{Data management: inventory}

Once the three-dimensional models and images of the tunnels were available, the element inventory could be started. All of this work was carried out in the office (Fig. 9).

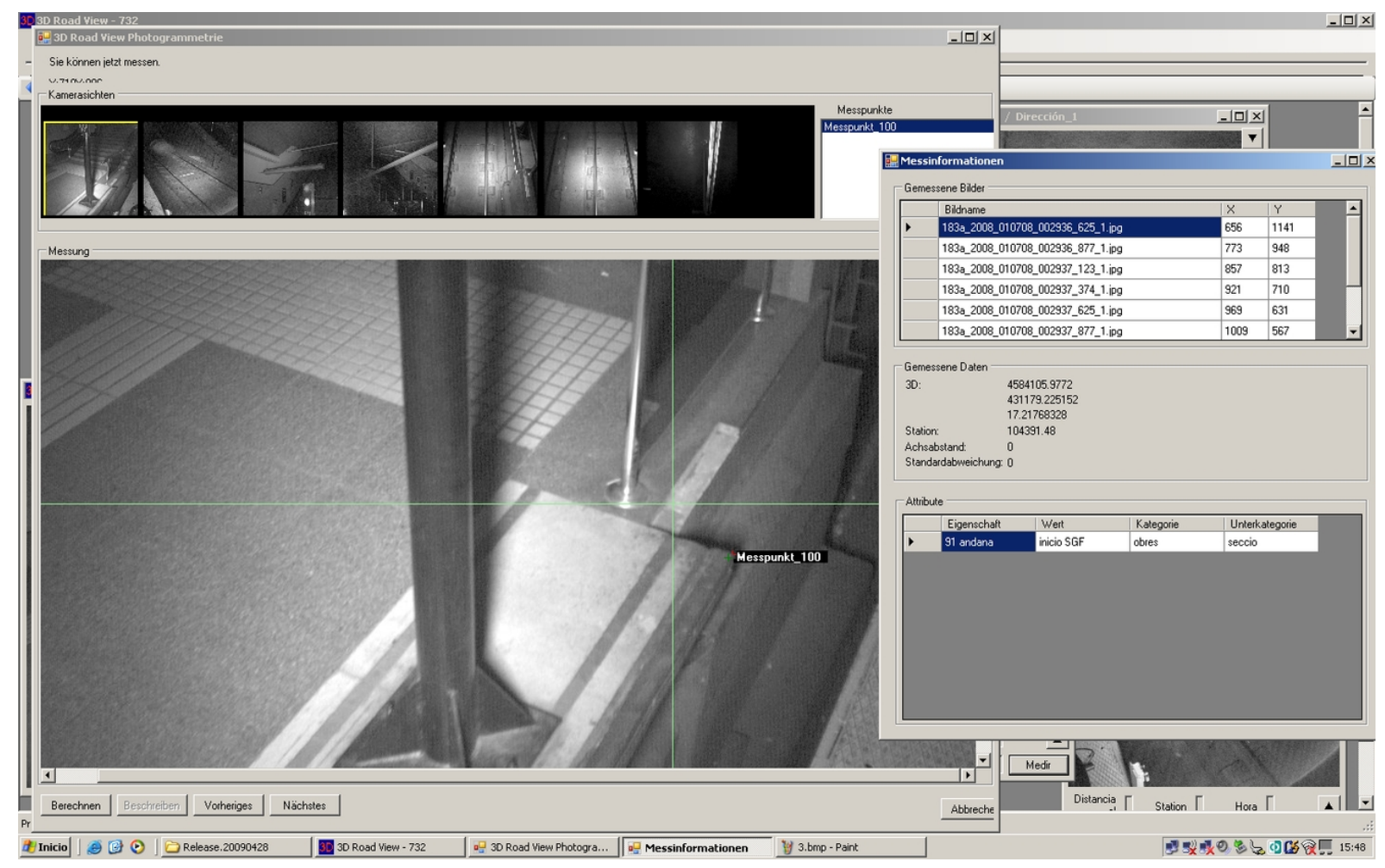

Fig 9. Element extraction for the catalogue from a stereographic pair

As a result of this phase, a list with the inventory of items in the catalogue was 
obtained, with over 200 different items established by the TMB.

\section{Analysis and results}

Since all the tasks to be performed are supported on the topographical network established in its three levels, the coordinates of the vertices that compose it must be precise enough.

The closures of the traverses of the tie network ranged from 0.001 to $0.006 \mathrm{~m}$, which in all cases, was below the established tolerance as a function of the technical characteristics of the topographic equipment and the length of the traverse. Therefore, the values were adjusted by least squares using the method of indirect equations, independently from the planimetry and altimetry. After adjustment, coordinates were obtained with an average $R M S$ of $1 \mathrm{~cm}$ in the three components. Any observable difference was rejected in the w-Baarda test.

The closures of the traverses of the Interior Network ranged from 0.002 to $0.09 \mathrm{~m}$, which in all cases was below the established tolerance. The adjustment method was the same as in the previous case. The average RMS obtained or the coordinates after adjustment was 1 $\mathrm{cm}$.

Data from photographs and scanning should be differentiated in the analyses of precision. The images provide information whose level of detail allows any object to be perfectly identified, while scanning provides continuous, but less detailed, information. It is not suitable for the identification of elements, but is perfect for understanding geometries and positions.

In order to test the reliability of kinematic surveys involving both photogrammetric and laser scanning, some well-defined elements were distributed throughout several areas of study. These were measured and their coordinates were compared with those obtained by 
kinematic methods. The results showed that there were discrepancies of centimetre order, which were within the precision required in all cases.

After several comparisons among coordinates of details from the point cloud and topography, the average discrepancy was estimated at $\pm 1 \mathrm{~cm}$, which is consistent with the precision of the systems and methodologies used. It should be noted that the greatest differences, which in no case exceeded a centimetre, were in areas, such as platforms, which were not available targets to adjust the trajectory.

The results obtained with the monoscopic or stereoscopic photogrammetric process showed values with a mean difference of $44 \mathrm{~mm}$ in planimetry and $56 \mathrm{~mm}$ vertically, in absolute values. In no case did the differences exceed $10 \mathrm{~cm}$. These variations are due to the computation of the position in indirect orientation of the cameras, since the error in photocoordinates can be considered residual.

The kinematic surveys resulted in a Database with the inventoried elements linked to pack images and the display module of the software, and the georeferenced point clouds. The volume of photogrammetric data was $430 \mathrm{~GB}$, with nearly three million georeferenced photographs (7 images for each $0.5 \mathrm{~m}$ section in each railway).

Thus, the identification, positioning and definition of the element attributes to the catalogue are carried out from the images, while data from the laser scanning were used to georeference the trajectory, and complement the interpretation of the different types of infrastructure, to obtain geometries. 


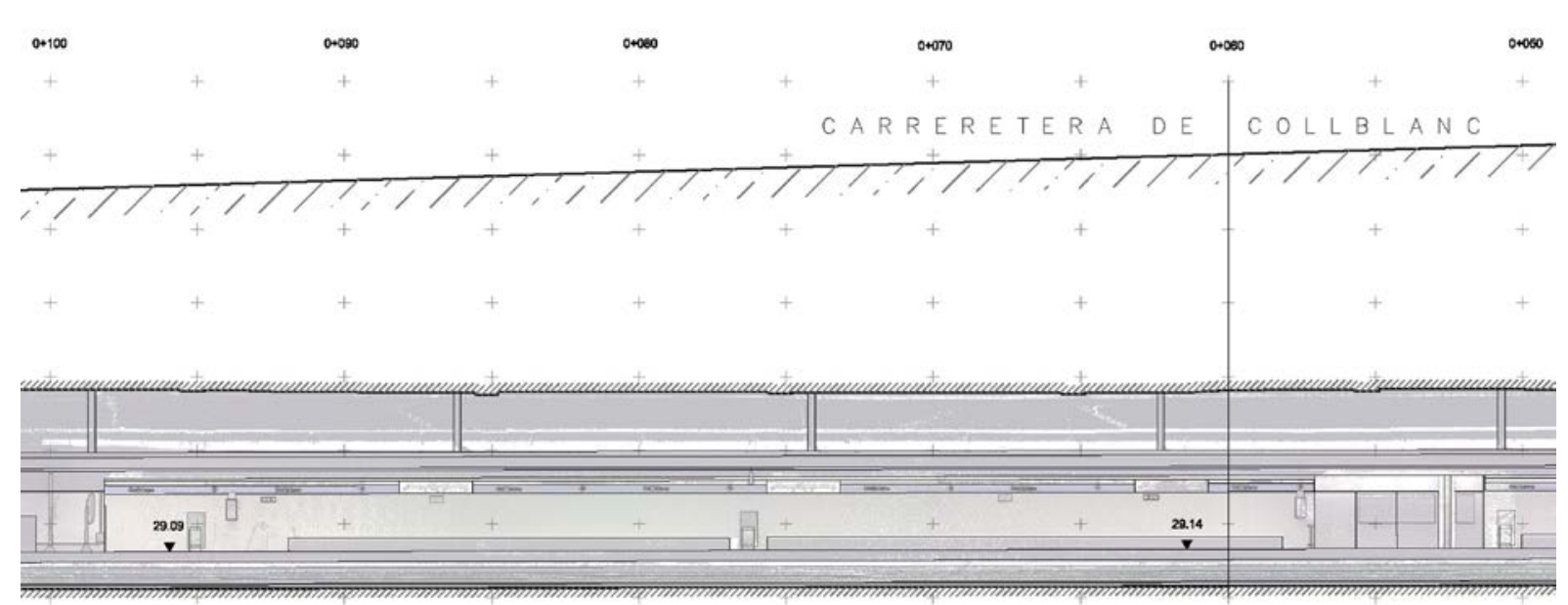

Fig 10. Platform longitudinal section

At present, data captured years ago can still be used to obtain information about sections (Fig. 10), planimetric maps and other features without having to go into the tunnel, since the original data are continuous and homogeneous in all the galleries.

\section{Conclusions}

The results show the working method as the best that offers the best binomial productivity/quality, taking into account the strict conditions of work in infrastructure of this nature.

The inability to directly georeference underground infrastructure and the fact that the working space is a linear area of reduced dimensions has meant that mobile mapping, the solution that has been adopted and widely used in urban surface surveys, is not directly applicable.

The duality of techniques available in these systems allows the creation of a $3 D$ laser scanner model, which can then be used in the office to obtain the geometry of tunnels, platforms and railroads with centimetre accuracy and precision. However, an inventory of all items in absolute coordinates and their attributes can be obtained from photogrammetric pairs. 
As the work can be done in the office, specialists in each of the categories do not need to enter the tunnels, and the costs and time are reduced.

In any case, the geodetic network must be of good quality to achieve the accuracy sought in other processes that require control points.

Table 1. Comparison between the traditional and new method

\begin{tabular}{|c|c|c|}
\cline { 2 - 3 } \multicolumn{1}{c|}{} & Traditional & New \\
\hline $\begin{array}{c}\text { Technic specialist in } \\
\text { field }\end{array}$ & 5 & 1 \\
\hline $\begin{array}{c}\text { Technic specialist in } \\
\text { office work }\end{array}$ & 5 & 5 \\
\hline Time in data capture & 6 months & 1 week \\
\hline Data capture type & Discrete & Continuous \\
\hline Use & Limited & Reused \\
\hline Future use & No & Yes \\
\hline Update & Yes & Yes \\
\hline
\end{tabular}

In the classical case of working in the tunnel gallery with various specialists, the hourly availability is limited to 4 hours in conditions of difficult access and low performance due to the environment (low illumination, long distances and extreme conditions, among other factors). In this case, only the operators are involved in the task. In the case of working with information extracted from the kinematic survey in which spatial information and images were captured, the data are available to all technicians 24 hours a day, at the same time, and in conditions of comfort and safety that are incomparable with the above method. In addition, the latter method allows new data to be extracted without accessing the galleries, Table 1. 
Once these georeferenced three-dimensional models of the infrastructure are available, a new task must be considered: updating the information. This requires systems that allow changes to be updated as they occur, without undertaking a complete survey (and thus saving resources). To undertake this task of updating the inventory, we believe that the best option is systems based solely on photogrammetry. Further research should be undertaken in this area.

\section{References}

Baarda, W., 1967. Statistical concepts in geodesy, Netherlands Geodetic Commission. Publications on Geodesy, New Series 2, 4.

Dai, F. and Peng, W.B., 2013. Reality capture in construction engineering applications using close-range photogrammetry. Applied mechanics and materials, 2795, 353-356.

Gikas, V. and Stratakos, J., 2012. A novel geodetic engineering method for the accurate and automated extraction of road/railway alignments based on the bearing diagram and fractal behaviour, IEEE transactions on intelligent transportation systems, 13, 115-126.

Gräfe, G., 2007. High precision kinematic surveying with laser scanners, Journal of Applied Geodesy, 1, 185-199.

Han, S., Cho, H., Kim, S., Jung, J. and Heo, J., 2013. Automated and efficient method for extraction of tunnel cross sections using terrestrial laser scanned data. Journal of computing in civil engineering, 27(3), 274-281.

Jacobs, G., 2005. High definition Surveying \& 3D laser scanning uses in transportation. Professional Surveyor Magazine, April.

Kingston, T., Gikas, V., Laflamme, C. and Larouche, C., 2007. An integrated mobile mapping system for data acquisition and automated asset extraction. In International Symposium on Mobile Mapping Technology.

Koch. C. and Brilakis, I., 2011. Pothole detection in asphalt pavement images. Advanced engineering informatics, 25 (3), 507-515.

McCarthy, T., Fotheringham, A.S., Charlton, M., Winstanley, A. and O’Malley, V., 2007. Integration of LiDAR and stereoscopic imagery for route corridor surveying. Mobile mapping technology, 37, 1125-1130. 
Pejic, M., Bozic, B., Abolmasov, B. and Gospavic, Z., 2013. Design and optimization of laser scanning for tunnels geometry inspection, Tunnelling and underground space technology, 37, 199-206.

Puente, I., González-Jorge, H., Martínez-Sánchez, J. and Arias, P., 2013. Review of mobile mapping and surveying technologies. Measurement, 46 (7), 2127-2145.

Puente, I., González-Jorge, H., Martínez-Sánchez, J. and Arias, P., 2014. Automatic detection of road tunnel luminaires using a mobile LiDAR system, Measurement. 47. 569-575.

Ratshidaho, T.T., Tapamo, J.R., Claassens, N. and Govender, J., 2014. An investigation into trajectory estimation in underground mining environments using a time-of-flight camera and an inertial measurement unit. South african journal of industrial engineering, 25(1), 145-161.

Roca-Pardiñas J., Argüelles-Fraga R., López, F. and Ordóñez, C., 2014. Analysis of the influence of range and angle of incidence of terrestrial laser scanning measurements on tunnel inspection. Tunnelling and underground space technology, 43, 133-139.

Scaioni, M., Barazzetti, L., Giussani, A., Previtali, M., Roncoroni, F. and Alba, MI., 2014. Photogrammetric techniques for monitoring tunnel deformation. Journal earth science informatics, 7 (2), 83-95.

Seo, D.J., Lee, J.C., Lee, Y.D., Lee, Y.H. and Mun, D.Y., 2008. Development of cross section management system in tunnel using terrestrial laser scanning technique, In: Proceedings of the international archives of the photogrammetry, remote sensing and spatial information sciences, XXXVII (B5), 573-581.

Slama, C.C., Theurer, C. and Hendrikson, S.W., 1980. Manual of photogrammetry $4^{\text {th }}$ Edition. Falls Church: American Society of Photogrammetry.

Talaya, J., Bosch, E., Alamús, R., Serra, A. and Baron, A., 2007. GEOVAN: The mobile mapping system from the Cartographic Institute of Catalonia. In: Advances in Mobile Mapping Technology. London: Taylor \& Francis Group.

Velasco, J., Herrero, T. and Prieto, J., 2014. Metodología de diseño, observación y cálculo de redes geodésicas exteriores para túneles de gran longitud. Informes de la Construcción, 66 (533).

Velasco-Gómez, J., Prieto, J.F., Molina, I., Herrero, T., Fábrega, J. and Pérez-Martín, E., 2016. Use of the gyrotheodolite in underground networks of long high-speed railway tunnels. Survey review, 48, (350).

Wang, T.T., Jaw, J.J., Chang, Y.H. and Jeng, F.S., 2009. Application and validation of profile-image method for measuring deformation of tunnel wall. Tunnelling and underground space technology, 24(2), 136-147. 
Yoon, J.S., Sagong, M., Lee, J.S. and Lee, K.S., 2009. Feature extraction of a concrete tunnel liner from 3D laser scanning data. NDT\&E international, 42(2), 97-105.

Zhu, Z. and Brilakis, I., 2009. Comparison of optical sensor-based spatial data collection techniques for civil infrastructure modeling. Journal of computing in civil engineering, 23 (3), $170-177$. 